\title{
Information Demand Pattern for Teams
}

\author{
Dirk Stamer, Kurt Sandkuhl and Veronika Zeiner \\ Rostock University, Institute of Computer Science, Albert-Einstein-Str. 22, 18059 Rostock, Germany \\ \{dirk.stamer, kurt.sandkuhl, veronika.zeiner\}@uni-rostock.de
}

\begin{abstract}
Modern organizations face the challenge of having to manage an increasing amount of information. The resulting information overload leads more and more to problems in decision making with potentially negative economic consequences. Decision-makers and knowledge intensive workers are especially affected. To address this problem, information demand patterns were proposed which capture organizational knowledge about the information demand of single roles. This work extends the concept of information demand patterns from single roles to teams. Using the knowledge intensive field of project management, the paper shows how to apply the concept of information demand patterns for a whole team. The contributions of this work are (1) the methodical approach to develop information demand patterns for teams, (2) an actual information demand pattern for a steering committee in the context of project management, (3) reflections on the differences between role patterns and team patterns.

Keywords: Information logistics, information demand analysis, information demand pattern, team information demand pattern, information overload, project management.
\end{abstract}

\section{Introduction}

Every day a lot of new information is produced in publicly available media, social networks and organizations' internal sources. The overall volume of information has significantly increased in recent years. Society has changed to an information society, where the shortage or missing availability of information cannot be viewed anymore as an obstacle for a demand-oriented information supply - finding the right information by the person who needs it becomes a more challenging task. This applies not only to society at large; organizations also are faced with an ever-growing amount of information [1] and the challenge to supply demand-oriented information to decision makers and organizational workers. Information overload has proven to be of a high topicality as recent studies have shown [2].

Contrary to earlier assumptions that more information is supporting better and more informed decisions and improves problem solving and decision-making processes, today the opposite effects can be observed. The amount of information is an increasing problem in today's information society [3]. Locating relevant information turns out to be difficult and consequently the requirements grow for a suitable structuring and a tailored access [4].

Information logistics, as an application field of business information systems, deals with this problem and aims to improve of the information flow in organizations by providing a demandoriented information supply. Information logistics offers methods, concepts and tools to achieve this improvement. The concept of demand-oriented information supply denotes the provision of 
the right information at the right time, in the right quality, in the right form and at the right place for the person needing the information [5].

In order to improve the information flow within companies information demand patterns (IDPs) were proposed [6]. IDPs specify the information demands of a single role ("role pattern") represented by a person within an organization to identify repeatedly required information for this role. As with patterns in other disciplines of computer science, these patterns have the purpose of capturing a proven solution to a problem in order to reuse it in other application scenarios. With information demand patterns, the identified organizational knowledge can be collected in a structured and reusable way that enables information systems to support the targeted role and counteract the flood of information. The general goal is to improve the efficiency of information provision; hence, work processes can be effectively designed and implemented. Furthermore, the time required for finding the relevant information can also be reduced. Moreover, according to objectives of information logistics [6], for the same role, it should be possible to provide the needed information on time and in the required quality and accuracy in different application contexts.

The organizational knowledge captured in information demand patterns can be used in practical information logistics solutions to reduce, for example, the information overload in email-communication, as shown by [7].

In the corporate environment, daily work is often characterized by teamwork in projects involving several participating roles. In the course of projects, which usually include new tasks and routine procedures, the above-mentioned problems relating to information supply can be observed. For each role in the project, team information overload is a potential problem due to the considerable amount of time used in finding relevant information. Simply reducing the supply of information is not a solution, since it obviously leads to the absence of the required information that may negatively affect the work [4].

Concerning teamwork, the question arises whether the concept of information demands patterns can be transferred from individual roles to teams in order to capture the information demands of the team and start the development of a generic solution for teams. A pattern, which describes the information demands of a whole team ("team pattern"), can help to improve structures and processes within the team and to generally increase the efficiency of the team, as it is developed on an aggregate level and has, therefore, a holistic perspective on the team. In the context of this paper, such a pattern is presented.

The following research question has been defined in this work: How can the concept of an information demand pattern for individual roles be transferred to a whole team? In addition to answering the research question, the identification of further research topics play a role in the context of information demand patterns for teams. This work can be seen as a precondition for developing a solution to reduce the information overload in working teams in organizations.

This work extends a previous work [8] and provides further insights regarding the presentation of the results, discussion of the results and further steps of investigation. The contributions of this work are: (1) the methodical approach to develop information demand patterns for teams, (2) an actual information demand pattern for a Steering Committee in the context of project management, (3) reflections on the differences between role patterns and team patterns.

The work is structured as follows: Section 2 describes related work and useful background information concerning information demand, information demand analysis, information demand patterns and project management. The methodical approach of the pattern development and the resulting team pattern for a Steering Committee are introduced in Section 3. Section 4 presents the approach and the results from validating the team pattern. Section 5 discusses observations from the development process and the relation between team pattern and role pattern. The conclusions and an outlook of future studies are given in Section 5. 


\section{Background}

Information demand analysis is a precondition for constructing an information demand pattern. Although the approach of Sandkuhl to develop an information demand pattern [6] strongly relates to the information demand analysis method by Lundqvist [9], we examined different methods of information demand analysis as well as areas of research in this field. Strauch and Winter [10] describe a detailed approach on information demand analysis concerning data warehouses. Starting with a target group analysis, the analysis leads to an information map, which will be transformed into a cube-structure model regarding several self-selected dimensions. Cappiello and Comuzzi [11] focused on an approach to define the optimal quality of information provided from an IT Service in order to completely satisfy user demand as well as the provider's organizational requirements. Further approaches optimize the process of identifying the information need by aggregating heterogeneous information sources and presenting the results to a user in a personalized manner [12].

This section briefly introduces key principles concerning information demand (Section 2.1), the method of information demand analysis used for determining information demands (Section 2.2), the concept of "information demand pattern" for single roles (Section 2.3) and selected aspects of project management required for developing the Steering Committee team pattern (Section 2.4).

\subsection{Information Demand}

In this work the understanding and definition of 'information demands' is based on the results of research that was carried out from 2005 to 2007 [9]. The main objective of the study was the identification of relationships between information needs and work-related aspects such as processes, resources and organizational structures. The investigation included interviews with 27 people from three different organizations. The interviewees came from both the management and operational functions such as production, product development and administration. The result of this work was, inter alia, a definition of information demands, which is used in the further course of this work.

Information demand is the constantly changing need for relevant, current, accurate, reliable, and integrated information to support (business) activities, whenever and wherever it is needed [13].

Figure 1 illustrates the concept of information demand and its relationships to other concepts such as role, responsibilities, tasks, source and information - in more detail. As introduced by Lundqvist, the role of a person in a company is the starting point for eliciting and capturing the information demands. A role filled by a person has various responsibilities, which are an essential part of defining the role. The responsibilities of the role define the tasks that have to be carried out by a role within a company. The tasks of a role, in turn, define the information demands essential to ensure that the role can perform its duties. These information demands can be satisfied by information; this information is used by the role to perform its duties. Defined sources providing this information are used by roles to satisfy their information demands [13].

\subsection{Information Demand Analysis}

Information demand analysis is an elicitation and modeling method which allows a systematic analysis of information demand [4]. The information demand is identified in a process consisting of several phases, which can also be considered as method components. The phases are listed and their contents [14] are briefly described below.

- Scoping: In the first phase, the area of analysis is defined. More concretely, the part of an organization to be analyzed with respect to the information demands of roles is selected 
and informants who can provide the required information during the actual analysis activities are identified. Furthermore, the observed problems are discussed with informants in order to motivate them to actively contribute to the analysis.

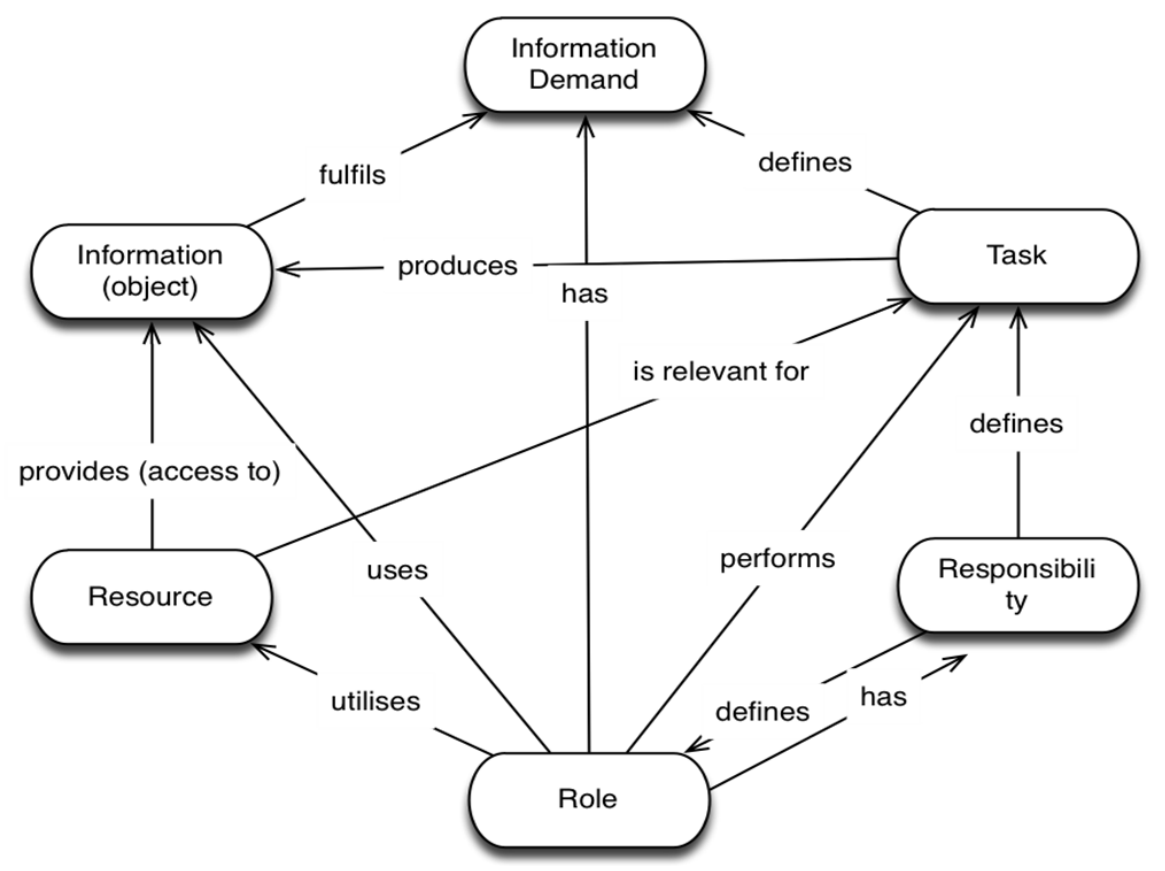

Figure 1. Information Demand and related concepts (based on [13] and [14])

- Information Demand Context Modeling: Based on the defined scope, the basic information demands for all organizational roles in the scope are identified. For this purpose, the responsibilities and tasks of each role are identified and the information required for fulfilling the responsibilities is captured. Furthermore, the relationships between roles, tasks, resources and information have to be considered. The result of this step is called the information demand context. It is assumed that all people with the same role have the same information demand for a specific task. This correlates to the rolecentric perspective.

- ID-Context Analysis and Evaluation: In the third phase, the information demand (ID) contexts for each role, namely, the results from the previous step, are analyzed with respect to improvement requirements. Such requirements could be the resultof situations such as: certain tasks having missing information, superfluous information that should no longer be provided, wrong points in time of information provision or the inadequate quality of information that has to be adjusted. The analysis results are documented and presented in a suitable format (e.g., in a modeling language) as a basis for further work. This information will be further classified and refined and could be linked to already existing information demand patterns in the company.

- Representation and Documentation: Finally, the previously created models and documents, which are in different notations, are summarized and in a consistent and coherent form. This involves a continuous and iterative process until the goal of the analysis is achieved.

Figure 2 illustrates the method of information demand analysis according to Lundqvist [14]. 


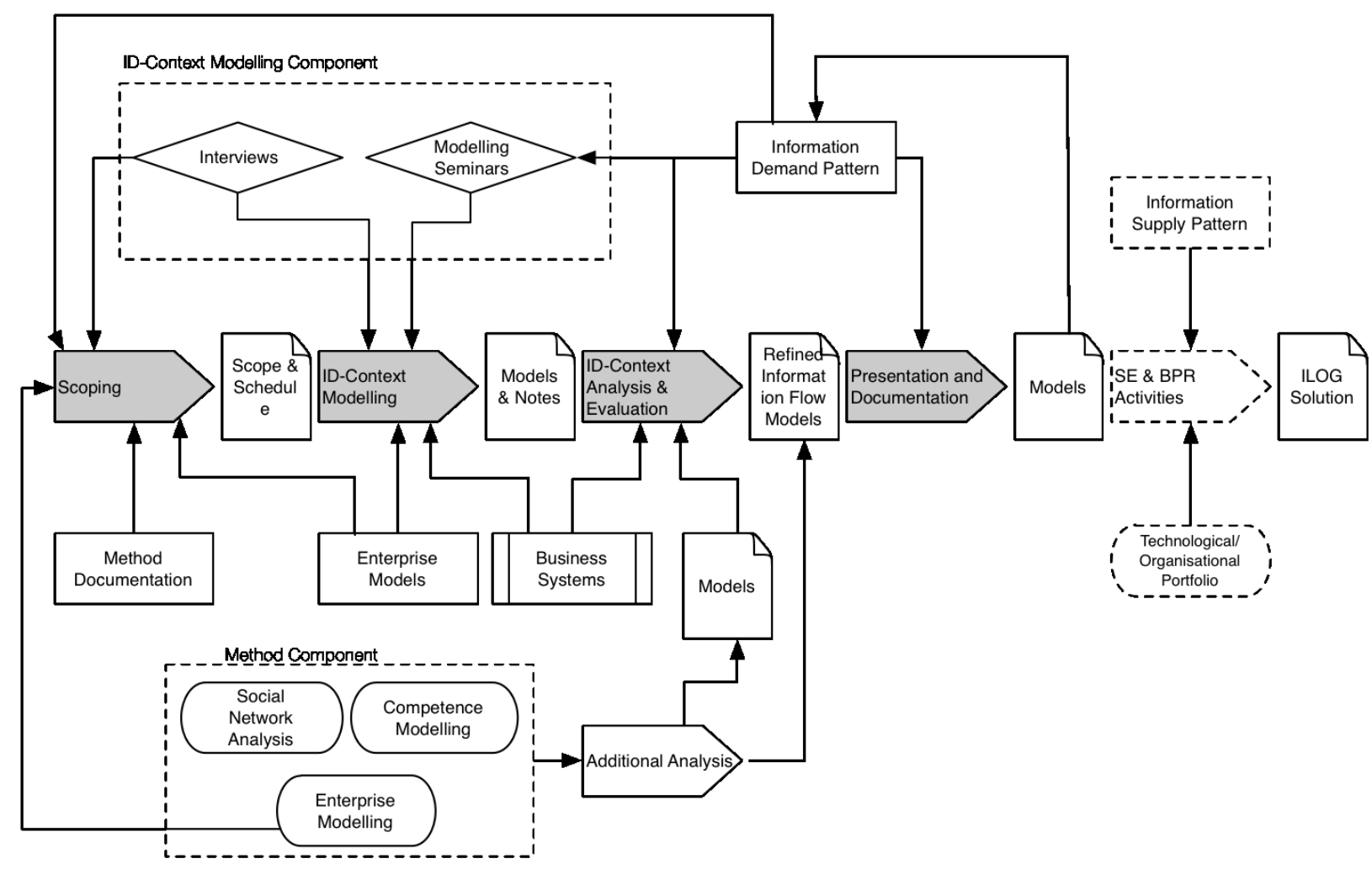

Figure 2. Overview to information demand analysis method (based on [14])

\subsection{Information Demand Pattern}

Based on the conceptualization of the information demand of a role in a company (see Section 2.1), Sandkuhl proposed the concept of an information demand pattern. Similar to patterns in other disciplines of computer science, information demand patterns have the purpose of capturing a proven solution to a problem in order to reuse it in other application scenarios. With information demand patterns, the identified organizational knowledge is collected in a structured and reusable way. The term "information demand pattern" is defined according to [6] as follows: An information demand pattern addresses a recurring information flow problem that arises for specific roles and work situations in an enterprise, and presents a conceptual solution to it.

Information demand patterns consist of five integral components: Name of the pattern, Organizational context, Problems, Conceptual solution and Effects.

The name is used to identify the pattern. This is usually the name of the role, which the pattern describes.

The organizational context explains the application context in which the pattern can be used. This can be departments, functions or domains.

Problems represent the difficulties and challenges that a person faces in fulfilling his/her role in the company. Furthermore, duties and responsibilities of the role are subsumed under this point.

How the described problems of the role can be solved is shown in a conceptual solution. The conceptual solution is divided into three areas: information demand, quality criteria and timeline. Information demand describes the information necessary to fulfill the duties and responsibilities of the role. The quality criteria describe the quality of the information that must be available, such as the general importance of the information as well as its accuracy, timeliness and completeness. 


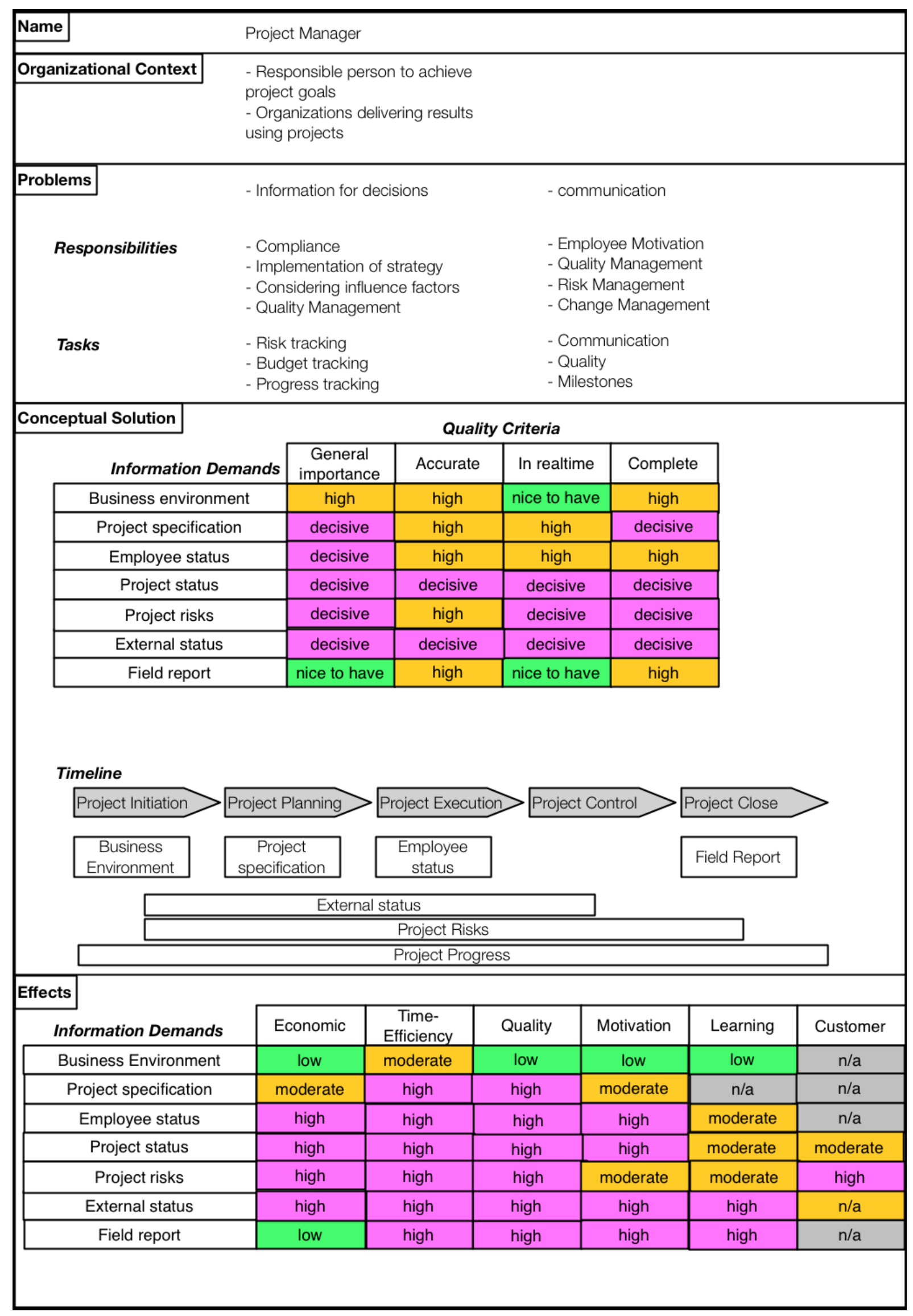

Figure 3. Overview to information demand pattern "Project Manager" 
Figure 3 shows an example of an information demand pattern (IDP) by summarizing the essential content of the IDP for the role "project manager". The information demands may have the values 'decisive', 'high' or 'nice to have'. If an information demand has the value 'decisive', this means that the role cannot be carried out without the information. If the value of the information demand is 'high', the role can be accomplished, but the quality will suffer. If the value is just 'nice to have' the role is able to accomplish the task in the desired quality, but having the information on time may increase the quality.

The timeline represents the time at which the required information must be available, at the latest. In the example (Figure 3) the timeline of the demand pattern was structured similarly to the usual phases of a project.

The effects part describes the effects that may occur if the information is not available or not available on time. The possible effects occurring may be associated with the following dimensions: economic effects, time and efficiency, quality of work, motivation, learning, experience and customers [6].

The concept of information demand pattern for single roles has been studied in several other works and its applicability has been validated [15], [16], [17].

\subsection{Project Management}

The German Institute for Standardization (Deutsches Institut für Normung - DIN) defines a project by the uniqueness of its conditions, such as targets as well as temporal, financial, personal or other limitations, and by the manner of organization specific to the project (DIN 69901) [18], [19]. A project is, therefore, characterized by an approach that is different from daily work. This approach is structured and performed separately from the actual organizational structure. Other characteristics of a project are the time limitation and the limited resources.

Furthermore, the project management is defined as "the totality of leadership, organization, techniques and agents for the initiation, definition, control and completion of projects" [18], [19]. In order to reduce the complexity of projects, the project management divides a project into phases. Project phases are sections in a project where additional control is needed to effectively manage its completion. Project phases are typically completed sequentially, but can overlap in some project situations [20].

The DIN differentiates between project management phases and project phases. In this sense, the project phases are special sections that are adapted to the activities in an organization. The project management phases are based on the segmentation of the general activities in project management. For this, a classification into five phases is done: initialization, definition, planning, management and completion [18], [19].

There are several well-established standards, which can be used for daily work dealing with projects. Standards for project management include the previously presented concepts and structures and try to create a unified understanding of the concept's extension so that further aspects, such as roles, processes and methods, can be described [21].

Some standards have gained international importance and are used in practice in many companies. In particular, these are: the Project Management Body of Knowledge (PMBOK), Projects in a controlled environment (PRINCE2) and the IPMA Competence Baseline (ICB) of the International Project Management Association [22].

These well-accepted standards are used later in this work in order to identify relevant roles that are typical part of a project. 


\section{From Role Pattern to Team Pattern}

The background for our research described in Section 2 shows that previous research work focused on understanding the information demand of single roles (Section 2.1), analyzing this demand (Section 2.2) and capturing it in patterns (Section 2.3). What has not been explored so far is whether or not the concept of information demand can also be applied for teams consisting of several roles and what structural differences between "role pattern" and "team pattern" would be required.

In order to address this issue, first, Section 3.1 discusses a methodical approach for developing the envisioned "information demand pattern for a team", hereinafter called team pattern. The purpose of a team pattern is similar to a role pattern: capturing organizational knowledge about the information demand of a team, which can be applied to establish a better information supply for the team. When developing the team pattern we assume that the information demand patterns for each role ("role patterns") in this team can form the basis of the team pattern. Hence, the method for information demand analysis can also be applied for team patterns by analyzing the information demand of all roles in the team. Second, Section 3.2. addresses the actual development of the team pattern using the methodical approach. The team pattern structure and the actual pattern of project management by the team's "Steering Committee" are described and discussed there.

\subsection{Methodical Approach}

Sandkuhl [6] presented an approach for the development of information demand patterns in general and for specific organizational roles. The approach describes an iterative multi-step process, which is used to develop the team pattern and shown in Figure 4.

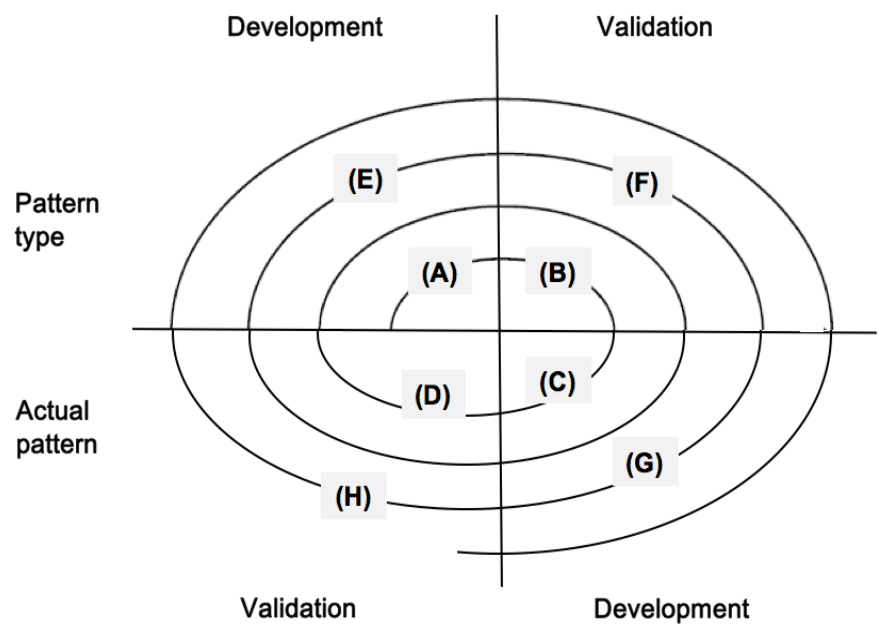

Figure 4. Development process of the team pattern

The first four steps (A)-(D) represent the first iteration, which is characterized by the participation of the research team in the development only. In the first step, the initial structure of the pattern is noted. In the second step, the suitability of the description of the information demands of an organizational role is checked. The structure is also checked for internal consistency and correctness. The third step involves selecting a simple but sufficiently complex organizational role and then developing the pattern content for the selected role. In the fourth step these are validated by their internal consistency and, finally, completeness is checked.

The second iteration circle includes the development and validation of the pattern with actors beyond the research team. It begins with the fifth step in a further elaboration of structure based 
on experience regarding the development and validation of the initial pattern (step (C) and (D)). Improvements concerning the structure and the pattern are described in the sixth step. The validation of the improved version now involves more actors as well. The iteration steps after the eighth step contain further elaboration and validation activities that involve the participation of stakeholders outside the research team and Academia [6].

Based on a literature analysis of the standard project management literature listed in Section 2.4, four roles were identified, which usually form the body of a Steering Committee according to PMBOK, PRINCE2 and ICB. These are: a project manager, a project sponsor, customer representatives and supplier representatives. It must be considered that, in addition to the four mentioned, there may be other roles attributed to the Steering Committee. For instance, Murray [23] added the role 'project assurance'. For the sake of generality, in the team pattern for the Steering Committee we included only the roles that occur in PMBOK, Prince2 and ICB.

Firstly, for each role, a single information demand pattern was developed. For this purpose, the standard literature for project management was searched for the duties and responsibilities of these roles.

After this the first substantive elaboration, a check was performed on whether the information demand patterns was consistent and complete. For this purpose, the information from the literature was further researched, and the pattern with the experience gained on consistency and completeness was checked. The identified improvements were thus incorporated into the information demand pattern.

Based on the improved information demand patterns, a validation was performed in the form of semi-structured interviews with representatives of the considered roles. This included representatives from the governing body of a university.

During the interviews, respondents were first asked openly about their duties, responsibilities and the resulting information demands. This was done without giving predefined response options in order to be able to recognize new aspects and afterwards to include them in the patterns. After the open survey, respondents were explicitly asked for information on the duties and responsibilities previously identified in the literature, in order to check the practical relevance of this information.

From the validated information demand patterns for each single role of the Steering Committee, a generally applicable information demand pattern for teams was developed, covering the team as a whole. From the information demand patterns for the team, all components of the individual roles can be derived. In addition, the team contains pattern elements that are relevant for the team as a whole. The information demand patterns of each role are validated through the semi-structured interviews.

\subsection{Information Demand Pattern for a Team}

The team pattern, which was developed using the methodical approach introduced in Section 3.1, will be presented in this section. This pattern is defined for the team "Steering Committee" and is illustrated in Figure 5. The team pattern is structured according to the structure of single information demand initially proposed by Sandkuhl [6], shown in Section 2.

The context of this information demand pattern is a team pattern for the Steering Committee of a project in an organization. The scope is the field of project management and refers to large and medium-sized organizations, although it can also be applied to small enterprises.

The project management deals with the planning, delegation, monitoring and control of all aspects of a project, and the motivation of all those involved; the objective is to achieve the project outcome within the expected performance targets in terms of time, cost, quality, scope, benefits and risks [24]. The Steering Committee is responsible for the success of the project and it takes on the direction and control of the project within its area of responsibility [24]. It consists of an executive (i.e., the chairman of the project board), a project manager, a user and a supplier representative [24]. 


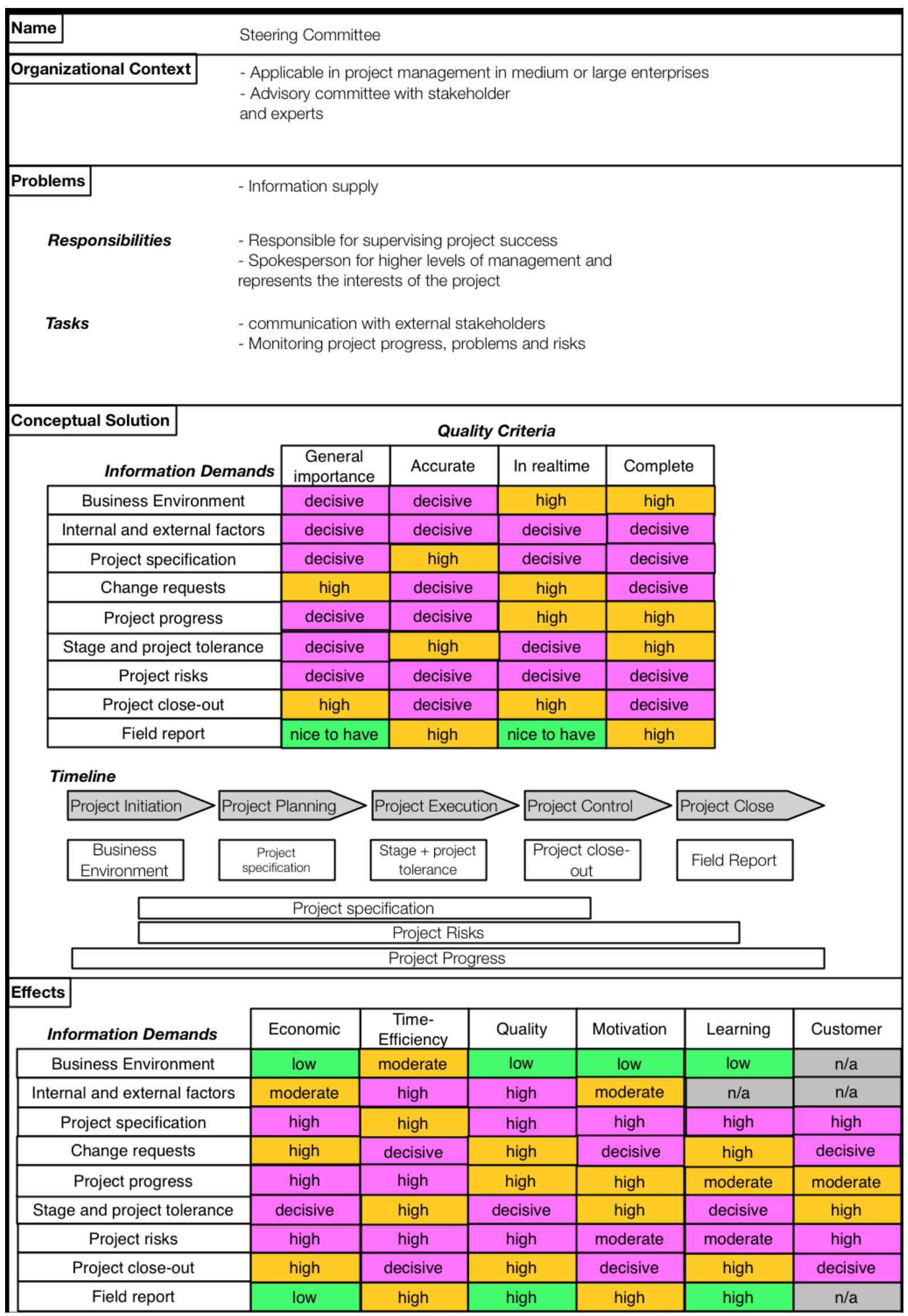

Figure 5. Overview to information demand pattern for team "Steering Committee"

The Steering Committee serves as a spokesperson for higher levels of management and represents the interests of the project to get support for the project and to sponsor the project. In 
addition, the project board is responsible for communication with the stakeholders outside the team. Within the scope of the provisions of the corporate management or program management, the Steering Committee takes over the overall responsibility and authority for the project. Furthermore, it is responsible for the provision of the financial resources and payments in kind [20].

The problems and the fields of activity that confront the Steering Committee are diverse and have their causes in both project internals and externals and can affect all project aspects, since it is the Steering Committee that takes the responsibility for the results of the project. Moreover, it is dependent on information about the current progress of the project, as well as problems and risks that have emerged during the project. Since information about project progress can be detailed, care should be taken in determining which information is provided to the Steering Committee and under what prerequisites, so that appropriate decisions can be taken promptly and the subsequent measures be effectively planned and implemented. Meetings should take place with just enough frequency for the coordination efforts to remain within an acceptable level.

If the Steering Committee does not receive information about all essential project aspects, or even if it is delayed, problems can occur with the coordination and regulation of the project. The consequences of the mentioned problems and risks can have serious effects on the organization, which can lead to economic consequences and damaged reputations [6].

Next follows the conceptual solution of the pattern with the information demand, which is based on the tasks and responsibilities of the team. As the body responsible for the success of the project, the Steering Committee takes over many different tasks and responsibilities. In principle, the Steering Committee is responsible for the project in its entirety and takes over its leadership. The project board is supposed to represent the project and the stakeholders and to promote the whole project [20]. Additionally, it aims to ensure uniform and effective decision-making [22]. Furthermore, it is responsible for providing the required resources for the project [20]. To sum up, it takes over planning, control, coordination and monitoring, and supports tasks.

The general areas of the information demand of the Steering Committee cover legal, organizational and technical aspects, as well as information about changes in these aspects.

Besides that, some fundamental information about the project is needed. This information regards the aim and size of the project and is needed in order to let the project board decide on the approval or rejection of the project. At the end of every project phase, once again the Steering Committee checks the progress and financial viability of the project before deciding whether it will be continued or stopped.

Generally, the information demand of the Steering Committee can be categorized into the following four areas: Business environment, Project specification, Project progress and Project closure.

Quality criteria are also summarized in Figure 5. The information demands are displayed in the left column. The quality criteria are structured as follows: the general importance of the information, its accuracy, time aspects and completeness. Compared with the information demand pattern of the project manager shown in Figure 3, it can be seen that, on the one hand, a Steering Committee has more information demands than a project manager. On the other hand, it can be seen that the quality criteria of similar information demands differ. Taking the information demand "project risks" as an example, the quality criteria "accurate" is considered "high" for a project manager and "decisive" for the whole Steering Committee.

Effects arise when the required information is not available or not available on time. The effects are described textually in the lower table in Figure 5. The following examples analyze economic effects. The economic consequences arise from missing or incomplete information and lead to additional costs for the project. This can be the case if contractual agreements with the customer are not observed. Furthermore, risks and changes can lead to additional costs because more time and more resources are needed for the project. Besides that, deviations of project and phase tolerances accumulate into additional cost factors. A lot of harm can result from 
terminating the project for the enterprise. On the one hand, this harm can arise from a decline in sales volume and reduced income, particularly if the project result should be commercialized. On the other hand, relations with the customer can be negatively influenced and it can cause damage to their reputation [20]. Collectively, these damages can lead to an existential threat for the enterprise.

\section{Team Pattern Validation}

The focus of the validation activities performed was to make sure that team pattern and the information demands of the different roles included in the "Steering Committee" team (see Section 3.2) were suitable for practical use. Expert interviews were used to validate the team pattern. As the development of the pattern was primarily based on academic literature, experts were selected from industry to balance theory and practice. The interviews were guided by two questions:

1. From the experts' perspective, do the information demands in the role patterns and in the team pattern cover all information demands for Steering Committee work in a way suitable for use in practice?

2. What importance of the information demands and what potential effects of not providing the information do the experts see?

Five interviews were conducted. The interviews were based on a list of guiding questions, which included questions about the interviewees' duties and responsibilities in the context of Steering Committee work. Furthermore, the different kinds of information required to fulfil the interviewee's tasks and responsibilities were the subject of the interviews. This included questions about the importance of the different kinds of information and questions about the impact of delayed or missing information. For this part of the interviews the tabular representation from the information demand pattern structure was used (see Section 3.2) and either made the interviewees fill in a blank table or did this based on their instructions. Two researchers noted down the answers to the other questions of the respondents. In four out of five interviews two researchers were involved. The overall structure of the guiding questions and the interviews followed the outline of the information demand analysis user guide [14].

The interviewees were representatives of the following roles: the client, the project manager, the users' representative and the suppliers' representative of an internal university project. The project under consideration was the introduction of a campus management system in a university, more specifically, the introduction of a technically and functionally integrated webbased information system for all processes and structures within a university. The five interviewees were the project manager, a customer and a supplier representative, and two user representatives. On average, each interview lasted thirty minutes.

The explanation of team pattern validation is structured as follows: first, the content analysis of the interviews (Section 4.1) is described, followed by a discussion about threats to validity (Section 4.2).

\subsection{Content Analysis of the Interviews}

From a methodical perspective, the interviews collected qualitative data which were analyzed using Mayring's approach to qualitative content analysis [27].The approach includes 6 steps:

Step 1 is to decide on the material to be analyzed, which in this case consists of the notes that the researchers took during the five interviews with Steering Committee members. All members were specially trained and very experienced in their roles and can be considered as experts for the required tasks.

Step 2 is to make explicit how the data collection was arranged and prepared. The experts were selected from existing projects at the home university of the researchers involved. The 
interviews were conducted face to face in a period of two weeks. They were prepared through email and phone contact with the interviewees. During the phone contact, the purpose of the interview was explained and an overview of the content was given.

Step 3 is to make explicit how the transcription of the material has to be done. The material was analyzed step by step, following the rules of procedure for standardizing the material into analytical units of content. The units were tagged as either being specific for an individual role or for the Steering Committee as a whole.

Step 4 concerns the subject-reference of the analysis, i.e., it has to be verified that the analysis is connected to the concrete subject of the investigation. Subject-reference was implemented by (a) defining the interview guidelines based on the research questions and their sub-questions and (b) using the subjects of these sub-questions as categories during the analysis. The categories matched the structure of information demand patterns.

Step 5 recommends theory-guided analysis of the data, which is supposed to balance the fuzziness of qualitative analysis with theoretical stringency. We took the state of the art into account during both the formulation of the sub-questions and analysis of the material.

Step 6 defines the analysis technique, which in this case was content summary. This attempts to reduce the material and create a manageable corpus, which still reflects the original material. For this purpose, the text was paraphrased, then generalized, and subsequently abstracted and reduced.

For reasons of brevity, Table 1 presents only a small part of the result of Step 6. Out of 7 categories represented in the interview questions, those selected for representation in this paper were "responsibilities," "information demand," and "effects" . The same categories were also used for the individual roles. The responsibilities mentioned in the interviews support the argumentation in Section 3.2 regarding responsibilities in the team pattern (see Figure 5). The category "information demand", on the one hand, reflects the information demands in the role patterns and, on the other, the information demands in the team pattern (i.e., all statements of the experts), can be assigned to patterns and all pattern contents are represented in the statements. Furthermore, the interviewees also confirmed the suitability of the pattern structure. This is not explicitly reflected in the (paraphrased and reduced) statements, since the general and introductory part of the interview was not subject to content analysis. The number of effects found in the analysis is much smaller than in the patterns. This can be explained by the use of the blank tabular representation for capturing effects during the interviews. The tables were not part of the content analysis since they were a separate part of the notes. However, the tables were compared with the effect tables in the patterns and resulted in adjustments in the pattern.

Table 1. Result of the content summary step (excerpt)

\begin{tabular}{|c|l|l|}
\hline \# & \multicolumn{1}{|c|}{ Category } & \multicolumn{1}{c|}{ Statement } \\
\hline C 2 & Responsibilities & $\begin{array}{l}\text { Responsibility for project success; coordinate opinion of all stakeholders; decide on change } \\
\text { requests; supervise project manager; accept deliverables; spokesperson for management } \\
\text { perspective; highest quality assurance instance }\end{array}$ \\
\hline C 4 & Information Demand & $\begin{array}{l}\text { All important in the project; quality problems; changes in business environment; risk register; } \\
\text { risk development; contingency plans; functional specification; non-functional requirements; } \\
\text { change requests; test reports; economic situation; resource consumption; resource shortage }\end{array}$ \\
\hline C 7 & Effects & No effective control; late detection of risks; project failure; communication problems \\
\hline
\end{tabular}

The role patterns were revised according to the results from the interviews. This applies also for the team pattern, since the contents of the individual samples partly are represented in team patterns. However, the team pattern also contains other content that indirectly connects to an individual role in the team. This additional content could not be fully validated in the present study and will be the subject of future investigations. An example is the timeline, which is a combination of individual timelines. 


\subsection{Threats to Validity}

The early identification of threats to validity and actions to mitigate such threats can minimize the effect on the findings. Common threats to empirical studies are discussed in [28]. The threats to validity can be divided into four categories: construct validity, internal validity, external validity and conclusion validity. With respect to construct validity, the results are highly dependent on the people being interviewed. Only persons experienced in Steering Committee work will be able to characterize the information demand of Steering Committee membership. To obtain a high quality of the sample, only experts having worked in this area for some time and hence having the required background were selected. Another common risk in studies is that the presence of a researcher influences the outcome. Since the selected experts in the study and the research group performing the study have been collaborating for a while, this is not perceived as a significant risk. Furthermore, there is a risk that the questions of the interviewer may be misunderstood or the data may be misinterpreted. In order to minimize this risk, the interview guidelines were double-checked by another researcher and the interview notes were taken by two researchers, which allowed the researcher to compare the notes if portions seemed unclear.

Regarding the internal validity, a common risk is that the data collected in the interviews did not completely capture the view of the experts regarding the development process. However, this threat was reduced by breaking down the interview questions in sections covering the different aspects of the information demand pattern's structure. Thus, this threat to validity is considered being under control. A potential threat of the study regarding external validity is that the actual interviews were conducted with only five experts. In future work, a study will be conducted with more participants or with more Steering Committees and their members. With respect to conclusion validity, interpretation of data is most critical. To minimize this threat, the study design includes capturing the relevant aspects by different interview questions. For example, the information demand for the Steering Committee is the subject of questions and also the subject of the table on the effects of missing or late information. Another risk could be that the interpretation of the data depends on the researcher and is not traceable. To reduce this risk, data analysis was performed according to Mayring's approach.

In summary, actions were taken to mitigate the risks identified. From the author's perspective, this ensures an appropriate confidence level regarding construct and internal validity. Future work such as additional studies for Steering Committees or other teams will contribute to increased confidence levels regarding external and conclusion validity.

\section{Discussion}

During the development and validation of the team pattern for a Steering Committee in project management, several observations were made which will be summarized and discussed in this section. The first part (Section 5.1) concerns the development process described in Section 3. The second part (Section 5.2) addresses the relation between team pattern and role patterns; this part is a result of observations made during pattern development (Section 3.2) and validation (Section 4).

\subsection{Team Pattern Development Process}

In the approach chosen for the development of a team pattern, role patterns were initially created for each role that are part of the team. Then these role patterns were aggregated into information demand patterns for a team.

With regard to the quality criteria and effects, there may be information demands that exist in more than one role. For instance, a project manager and a vendor representative who are both members of a Steering Committee may have the identical information demand 'project 
specification'. However, the characteristics of quality criteria may differ, in spite of the identical information demand. Referring to the example of information demand "project specification" concerning the quality criteria "in real time", the project manager may have the value "critical" and the vendor representative only the value "high". When aggregating the information demands 'project specification' of two roles, the question arises how to solve the above-mentioned issue?

On the one hand, the higher value concerning the quality criteria could be selected: Consider a role with an information demand marked as "critical" and a different role marking the same information demand as just "high"; this leads to an information demand for the whole team classified as "critical". It can be assumed that with this approach many information demands could be classified as too important for the whole team.

On the other hand, it is conceivable to implement a mathematical approach to calculate the values of the quality criteria set out in the aggregation. However, this procedure involves the risk that individual information demands are not considered as important for the team as they are in reality.

The general development approach for a role pattern is suitable for the development of group patterns as shown above. The proposed approach of Sandkuhl [6] for developing role patterns was adapted for this work and embodied as described. At the selected development procedure, role patterns are first produced, which are then combined to form a team pattern. It is also possible to develop a team pattern directly. This could be achieved through group interviews. The need to proceed with the development iteratively is more pronounced in the development of team patterns. Therefore, it is generally advisable to extend the development approach for an information demand pattern for teams through more iteration.

As already mentioned, it can be shown that mapping the newly developed information demand pattern for teams on the proposed structure for individual information demand patterns leads to some difficulties. Therefore, the structure has to be extended in order to be able to take into account all new aspects. Possible extensions that concern, for example, the effects of missing or delayed information not only affect customers but also the team itself. It can be shown that there are information demands within a team pattern that cannot be derived from the aggregation of the information demands of the roles involved. These information demands are mainly derived from the primary tasks of the team. For the future use of information demand patterns for teams, it is therefore proposed that the structure be extended to be able to identify the sources of the information demands.

\subsection{Relation of Team Pattern and Role Patterns}

The identified relation between a team pattern and the containing role patterns is shown in Figure 6 . In order to limit the complexity of the figure there are only 3 role patterns illustrated. The circles 1, 2, and 3 represent the information demand of a single role contained in a team pattern. Each role has its individual information demand, which it does not share with others since the information demands are determined by the tasks and responsibilities of each role.

The intersections $(4,5,6)$ between the information demands of two roles show that roles may have a partially identical information demand. This may be the case if both roles have to perform a similar task in the corporate environment.

Furthermore, there can be an information demand, common to all roles, that is included in the information demand pattern for teams; for example, the room number of a regular team meeting.

As already described, a team inside an organization has an information demand that goes beyond the sum of the information demands of the individual roles. This information demand is characterized by the task and responsibilities of the team itself. 


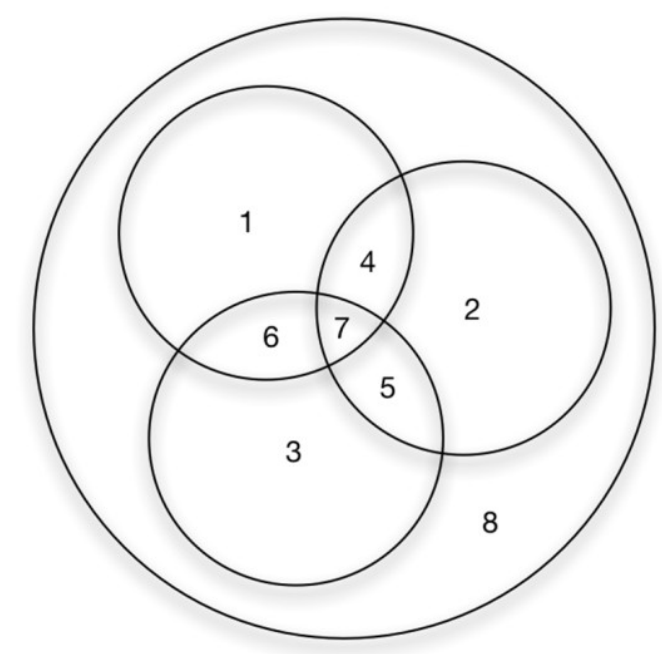

Figure 6. Relation between information demand of team pattern and role pattern

The question of whether a team pattern is more than the sum of its role patterns can be answered affirmatively. In particular, responsibilities and duties of the Steering Committee are quite different from the responsibilities and tasks of each role within the Steering Committee. This suggests that the information demands of a Steering Committee go beyond the information demands of each role as illustrated in Figure 6.

Furthermore, it has been shown that there are information demands within the team pattern which are created by the interaction of the roles involved. With regard to the structure of a group pattern, there is the need for action in order to reflect these additional information demands.

In this way the information, from the standards to roles and responsibilities, could be supplemented and revised. The information derived from these requirements could also be checked. In particular, the questions about the assessments at the time of deployment as well as the importance and the impact of information are an important part of the interviews. With the help of these interviews the verification and re-evaluation of patterns was possible.

For this purpose, the original classification of the author has been compared with those of the experts from the interviews. As the experts expressed their thoughts on the classification during the interviews, additional experiences and impressions were gained.

This is particularly important for the development of the team pattern, since this is based on the role patterns. It should be noted that only the parts of the team pattern could be examined, which result from the sum of the role patterns.

\section{Conclusion}

The work presented in this paper extended the work done concerning information demand patterns by adopting the structure of information demand patterns for single roles and extending it towards a team.

The case study used to develop the team pattern was a Steering Committee of a large project. As a starting point, the identification and definition of roles and teams in project management was required. For this purpose, three standards of project management were investigated to identify recurring roles in Steering Committees. On this basis, four information demand patterns for individual roles were developed and validated. Subsequently, one team pattern was derived out of the individual role patterns.

As a part of the validation of qualitative content, an analysis of semi-structured interviews with experts in Steering Committee work was conducted where at least one expert for each role of the selected Steering Committee was included. From this process, a team pattern composed of 
the contributions of individual roles and cross-team issues emerged. These cross-team aspects relate to the function of the team, in this case, control tasks and decision-making components. These team aspects cannot be expressed by the role patterns.

New perspectives in the development and application of information demand patterns resulted from the development of the team pattern and from the qualitative research approach. In this context, opportunities for improvements regarding the structure, content and the survey were identified so that future studies could build on it:

Rating scale: a suggestion for improvement relates to the different rating scales in the quality criteria and effects. A new uniform scale is proposed, where the distinction between the individual evaluation items is revised to facilitate the evaluation.

Quality criteria: Another way to improve aims at the quality characteristic "general importance". Since this is an abstract criterion and the classification is accordingly difficult, it is suggested that the classification of the other three criteria is derived. This could be implemented by calculating the median, where the median could conceivably be a weighting of individual classifications, for instance, to form the weighted arithmetic mean of it.

Information demand pattern for teams: As already mentioned in Section 4.1, a further validation of the pattern is needed. This might be done by an additional quantitative study.

Interview: While the information demand analysis was investigated and the results were validated several times, there is still room for improvement [17]. For instance, guidelines that are more concrete would be beneficial.

Furthermore, it might be interesting to evaluate other approaches to develop such a team pattern. This could consist of interviews with the entire team or indeed involve other forms of investigation, such as the observation of teamwork.

One useful application scenario could be the implementation of a team pattern in current research work, which aims at reducing the information overload in e-mail communication of teams by the information demand oriented filtering of e-mails using role patterns and recommendation systems [7], [25], [26].

Previous work has shown that information demand patterns for individual roles are well suited for the construction of systems, in particular, to address the problem of information overload in e-mail communication [7]. Since a variety of tasks in enterprises is accomplished in teamwork, it makes sense to transfer the concept of an information demand pattern for individual roles to a full team in an organization. The development of this information demand pattern for teams can therefore be considered as a previously missing piece of the puzzle in information logistics. The concrete development of a solution that uses information demand patterns can be seen as a contribution for practitioners. Through this work, the scientific body of knowledge has been enhanced by the concept of information demand patterns for teams. The aim of this work was also to show that the development of information demand patterns for teams is possible and to identify the necessary next steps of the research work, such as improving the structure of information demand patterns for teams. Further dimensions on a team pattern may include functions, behavior, structure, effects and the environment.

\section{References}

[1] A. Edmunds and A. Morris, "The problem of information overload in business organisations: a review of the literature," in International Journal of Information Management: The Journal for Information Professionals, vol. 20, no. 1, pp. 17-28, 2000. Available: http://dx.doi.org/10.1016/s0268-4012(99)00051-1

[2] P. Melinat, T. Kreuzkam and D. Stamer, "Information Overload: A Systematic Literature Review," presented at the 13th International Conference on Perspectives in Business Informatics Research, Lund, Sweden, vol. 194, pp. 72-86, 2014. Available: http://dx.doi.org/10.1007/978-3-319-11370-8_6

[3] H. Krcmar, "Informationsmanagement," Springer-Verlag, Berlin, Heidelberg, 2010. Available: http://dx.doi.org/10.1007/978-3-662-45863-1 
[4] L. J. Heinrich and D. Stelzer, "Informationsmanagement," 10th ed., Oldenbourg, 2011. Available: http://dx.doi.org/10.1524/9783486714593

[5] K. Sandkuhl, "Information Logistics in Networked Organizations: Selected Concepts and Applications," presented at the 9th International Conference on Enterprise Information Systems, Madeira, pp. 43-54, 2009. Available: http://dx.doi.org/10.1007/978-3-540-88710-2_4

[6] K. Sandkuhl, "Information Demand Patterns," presented at the PATTERNS 2011, The Third International Conferences on Pervasive Patterns and Applications, Rome, pp. 1-6, 2011.

[7] D. Stamer, A. Ponomarev, K. Sandkuhl, N. Shilov and A. Smirnov, "Collaborative Recommendation System for Improved Information Logistics: Adaption of Information Demand Pattern in E-Mail Communication," presented at the Proc. of the 7th International Workshop on Information Logistics and Knowledge Supply colocated with th International Conference on Perspectives in Business Informatics Research BIR, Lund, Sweden, pp. 35-48, 2014.

[8] D. Stamer, V. Zeiner and K. Sandkuhl, "Development and Validation of an Information Demand Pattern for a Team in Project Management," 2015.

[9] M. Lundqvist, E. Holmquist, K. Sandkuhl, U. Seigerroth and J. Strandesjo, "Information Demand Context Modelling for Improved Information Flow: Experiences and Practices," presented at the The Practice of Enterprise Modeling, Stockholm, 2009. Available: http://dx.doi.org/10.1007/978-3-642-05352-8_3

[10] R. Winter and B. Strauch, "Information Requirements Engineering for Data Warehouse Systems," New York, USA: ACM, pp. 1359-1365, 2004. Available: http://dx.doi.org/10.1145/967900.968174

[11] C. Cappiello and M. Comuzzi, "A utility-based model to define the optimal data quality level in IT service offerings," presented at the ECIS, 2009.

[12] A. Alhenshiri, C. Watters, M. Shepherd and J. Duffy, "Building Support for Web Information Gathering Tasks," 45th Hawaii International Conference on System Sciences (HICSS), pp. 1687-1696, 2012. Available: http://dx.doi.org/10.1109/hicss.2012.136

[13] M. Lundqvist, "Information Demand and Use: Improving Information Flow within Small-scale Business Contexts," Licentiate Thesis, Dept. of Computer and Information Science, Linköping University, Linköping, Sweden, 2007.

[14] M. Lundqvist, K. Sandkuhl, U. Seigerroth and E. Holmquist, "Infoflow. IDA User Guide. Handbook for Information Demand Analysis,” Jönköping, 2011.

[15] K. Sandkuhl and D. Stamer, "Towards Improving Structure and Content of Information Demand Patterns," presented at the 11th International Conference on Perspectives in Business Informatics Research, BIR 2012, Satellite Workshops and Doctoral Consortium, Nizhny Novgorod, 2012.

[16] K. Sandkuhl, "Improving Engineering Change Management with Information Demand Patterns," presented at the 8th International Conference on Poduct Lifecycle Management, 2011.

[17] L. Heinermann, D. Stamer and K. Sandkuhl, "Usability Evaluation of Method Handbook," presented at the 6th International Workshop on Information Logistics, Knowledge Supply and Ontologies in Information Systems (ILOG), Warsaw, 1st ed., pp. 11-22, 2013.

[18] Deutsches Institut für Normung, “DIN 69901-2:2009-01,”, Projektmanagement - Projektmanagementsysteme, 2009.

[19] Deutsches Institut für Normung, “DIN 69901-5:2009-01,”, Projektmanagement - Projektmanagementsysteme, 2009.

[20] Project Management Institute, "A Guide to the Project Management Body of Knowledge (PMBOK)," 5th ed., 2013. Available: http://dx.doi.org/10.1002/pmj.21345

[21] D. Ladwig, J. Kunze, M. Hartmann and P. Lang, "Exit matters - auf dem Weg in die Projektgesellschaft," Frankfurt: Lang, 2011.

[22] N. Ebel, "PRINCE2:2009 - für Projektmanagement mit Methode," Addison-Wesley, 2011.

[23] A. Murray, "Managing Successful Projects with PRINCE2," The Stationary Office, 2009.

[24] The Stationery Office Ltd, "Managing Successful Projects with PRINCE2," 2009.

[25] D. Stamer, "Reduktion der Informationsüberflutung durch Informationsbedarfsmuster in der E-MailKommunikation," presented at the Multikonferenz Wirtschaftsinformatik 2014, Paderborn, pp. 1226-1233, 2014. 
[26] D. Stamer, A. Ponomarev, N. Shilov and A. Smirnov, "Technical Perspective on Using Information Demand Pattern in a Collaborative Recommendation System for Improving E-Mail Communication,” CSIMQ, no. 2, pp. 31-45, 2015. Available: http://dx.doi.org/10.7250/csimq.2015-2.03

[27] P. Mayring, "Qualitative Content Analysis," Forum: Qualitative Social Research, 1(2), Art. 20, 2000. [Online]. Available: http://nbn-resolving.de/urn:nbn:de:0114-fqs0002204

[28] C. Wohlin, P. Runeson, M. Host, C. Ohlsson, B. Regnell and A. Wesslén, "Experimentation in Software Engineering: an Introduction," Kluver Academic Publishers, MA, USA, 2000. Available: http://dx.doi.org/10.1016/s0898-1221(00)90203-7 